\title{
SURVEYS OF X-RAY SOURCES
}

\author{
Herbert Friedman \\ (E.O. Hulburt Center for Space Research, U.S. Naval Research Laboratory, \\ Washington, D.C., U.S.A.)
}

My assignment this afternoon is to review briefly the status of the observational work that has been accomplished since the last IAU discussion of X-ray astronomy which took place at the Liège Symposium 3 years ago. Essentially, the methods of observation have not changed in any significant way. All of the information has come from small rockets, on the order of the Aerobee and the Skylark in size, and from packages carried on balloons. The quality of the surveys varies according to the objectives of each experiment. For example, my colleagues and I, at the Naval Research Laboratory, have attempted to perform broad-sky surveys to fill the picture of the general distribution of sources in space and, as a result, have had to sacrifice precision in position and high resolution. At the other extreme, Dr. Giacconi and his colleagues, at American Science and Engineering, Inc. and the Massachusetts Institute of Technology, have concentrated the available flight time on individual sources and have achieved remarkably high positional accuracy for some of the stronger sources - sufficient to associate them with optical objects. Dr. Fisher and his colleagues at Lockheed have taken an intermediate approach and used fan beam scans of limited regions of the sky, which give more precise position and better resolution in one dimension than the broad NRL surveys.

The broad-sky surveys that have been conducted by the NRL group use a largearea detector $\left(\sim 1000 \mathrm{~cm}^{2}\right)$ baffled by a honeycomb which offers an 8-degree field of view at half maximum and a triangular transmission pattern. Such surveys along the galactic equator in the general direction of the galactic centre indicate so many sources that it becomes very difficult to resolve the envelopes of unresolved sources into their discrete components, and much subjective judgment enters into the analysis. Recently, Dr. Giacconi and his colleagues have scanned the galactic centre region with much higher resolution. Essentially, I believe that they find all of the sources listed in the earlier NRL surveys and several additional sources.

The obvious thing about a map of sources is that most are positioned close to the galactic equator. There seem to be two major concentrations, one in the general direction of the galactic centre and the other in the general region of Cygnus and Cassiopeia. In a 1965 survey, we detected two high-latitude signals: one was in the vicinity of $\mathrm{M} 87$ with an uncertainty of about $1.5^{\circ}$, and the other source was in Leo and is designated Leo XR-1. With a distribution of this type, one can attempt some

Perek (ed.), Highlights of Astronomy, 180-183. (C) I.A.U. 
crude statistical analysis. The average displacement of 'galactic-centre' sources from the galactic equator is about $3.5^{\circ}$. The average separation of Cyg-Cas sources from the galactic equator is about $7^{\circ}$. If we assume that these are distributed like the general distribution of stars, with a mean separation from the galactic plane of about 150 parsec, we may estimate distances for these two distributions. The galacticcentre cluster appears to be about 2500 parsec distant and the Cyg-Cas group about 1300 parsec. These results suggest that the galactic-centre sources lie predominantly in the Sagittarius arm of the Galaxy and the Cyg-Cas sources in the Cygnus/Orion arm. However, when one looks at the data in more detail, there are discrepancies with such an analysis. There are evidences of either differences of spectral composition of the sources or in interstellar absorption. If the differences are attributed to interstellar absorption, then they either do not lie in the same arm or the interstellar gas is highly non-uniform. Dr. Giacconi's group has reported evidence for spectral differences between the galactic-centre group and the Cyg-Cas groups which may indicate that the latter group is much closer.

From comparisons of surveys made by different groups at different times, one finds evidences of differences in flux, from which we may conclude many of the sources are highly variable. In some cases the evidence is clear-cut as, e.g., when the observations have been made by the same group using essentially the same equipment at different periods of time. Where the observations have been made by different observers, there is always the problem of intercomparing the absolute fluxes observed when there are great uncertainties in the spectrum and these are reflected in the conversions to true fluxes with different techniques of observation.

During the past year, the AS\&E group have conducted a detailed survey of the collection of sources in the Sagittarius region and also in the Cygnus region with higher sensitivity and higher positional definition than the earlier surveys. By offsetting pairs of detectors and comparing the signals, they gained evidence for the position of the source along the direction of the slit pattern. In a typical experiment they have also scanned sources with a fan beam in two directions to obtain precise position. In Cygnus, they found that, where the NRL group had initially observed the sources Cyg XR-1 and Cyg XR-2, there certainly are strong sources, and their positions are only slightly displaced from the centres of the NRL uncertainty diagram. In addition, two other sources were found which were not picked up in the NRL surveys, but what is most important, where the NRL survey of 1965 showed a source at the position of Cyg A, they found no evidence within a factor of 10 of the flux claimed by the NRL group. From the fact that the NRL and AS\&E positions do agree so well on sources like Cyg XR-1 and Cyg XR-2, there can be little doubt about the solution of the aspect problem in the earlier NRL survey and the existence of a source in the direction of Cyg A. The fact that the Cyg A source did not appear a year later in the AS\&E survey must mean that there was a source there originally in 1965 , which declined in flux to the point where it was not observable a year later. Whether the 
source was Cyg A or some galactic object is, of course, not indicated by these observations.

In April 1965 the NRL group found a signal which roughly fit the position of M 87. In order to substantiate or improve the evidence for the existence of such a source, in May 1967 the NRL group used a stabilized rocket which was programmed to do a very slow scan of a region about $14^{\circ}$ long, across Virgo, so that it would pass through the positions of $3 \mathrm{C} 273$ and M 87. The field of view was a fan beam, $1^{\circ}$ in the scan direction by $8^{\circ}$ in the direction normal to the scan. Position was determined by photography of the star field during the course of the flight. We devoted the entire time of the Aerobee-rocket flight to cover this small region and thus gain the maximum statistical accuracy and the greatest sensitivity of detection for this resolution. Unfortunately, the flight was a little low and the scan was terminated just slightly past the peak of the signal from M 87. The rocket was already so low in the atmosphere that an absorption correction due to atmospheric attenuation had to be applied. What the record shows are signals from the intersections of the fan beam with the directions of 3 C 273 and of M 87 within a tenth of a degree, which was the accuracy of the position measurement of the survey. In addition, there are three other sources which appear at about the $3 \Sigma$ level and they do not coincide with any well-known radio sources or quasars. To compare this result with the observation of 1965 , which was made with a coarse collimator, one has to recognize that the coarse collimator covered so large a field of view that it would have lumped together the M 87 source and all the others. When we take account of the broad field of view, the signals observed agree within the statistical error of the measurements.

There are many important questions that concern the 'diffuse' X-ray background. Is there a truly diffuse background? Is the flux consistent with the interaction of cosmic rays in meta-galactic or intergalactic space with the $3^{\circ} \mathrm{K}$ background, producing X-rays by inverse Compton scattering, or is it simply the integral of radiation from all galaxies? I think that from the observations made thus far one cannot decide how much of the background is diffuse and how much is unresolved discrete sources. Having improved the resolution substantially in the May 1967 survey of Virgo, we find more discrete sources, but the resolution must be much further improved before one can interpret the nature of the background.

With regard to spectra, the source which has been most frequently studied and for which the best data exist is Sco XR-1. I believe that the speakers who follow will go into detailed discussion of the significance of these data. A fairly good fit can be made with a bremsstrahlung distribution of $5 \times 10^{7} \mathrm{~K}$ except for a flux measurement near $50 \AA$ by the NRL group. Šklovsky has presented an interesting accretion model which takes into account that measurement as well as the measurement at higher energy. It is urgently necessary that the experimenters redetermine, with high quantitative accuracy, the flux of long wavelengths.

With the techniques that have been used in the observations that have been dis- 
cussed so far, one can hopefully go quite a bit further. For instance, Šklovsky has argued the importance of studying the Centaurus A source, which is a powerful radio galaxy, so close to us and therefore so widely distributed in angle that even with $1^{\circ}$ collimation one could separate fluxes from the radio plasmons, and from the optical region at the centre of this radio galaxy. The flux which was observed from the direction of $3 \mathrm{C} 273$ is about $2 \times 10^{-10}$ ergs $\mathrm{cm}^{-2} \mathrm{sec}^{-1}$ in the $1-10 \AA$ range and, theoretically, one might expect a flux very close to that from the radio plasmons of Centaurus $\mathrm{A}$, as a result of the scattering of the relativistic electrons on the cosmological background radiation.

I would like to just briefly mention what might be done by the time of the next IAU Assembly if sufficient priority were given to the conduct of X-ray astronomy. In the U.S.A. there are opportunities to take advantage of Apollo-system hardware, and designs have been made to carry very large area detectors in such a system. Plans are being developed to carry up to 100 square feet of X-ray detector. With such a large aperture it would be possible to produce an X-ray catalog of sources, that would be analogous to the $3 \mathrm{C}$ catalog of radio sources, in the time of one flight. Some experimenters are concentrating on the development of large mirror systems. Dr. Giacconi's group in particular have been trying to promote opportunities for experiments with a very large reflecting telescope which would offer both high sensitivity and high resolution for studies of discrete sources. All of these things are technically possible and may come to pass sooner than we think.

\section{DISCUSSION}

S.I. Syrovatsky: What is the angular resolution of the large array of detectors which you mentioned at the end of your paper?

H. Friedman: $1^{\circ}$ by $4^{\circ}$.

R.J. Weymann: What is the flux from $\mathrm{M} 87$ ?

H. Friedman: The flux from M 87 is $8.7 \times 10^{-10} \mathrm{ergs} \mathrm{cm}^{-2} \mathrm{sec}^{-1}$ in the $1-10 \AA$ band if we assume a power law spectrum of index 1 , we would get roughly the same result with a bremsstrahlung spectrum of $5 \times 10^{7} \mathrm{~K}$. That would make the luminosity $1.1 \times 10^{43} \mathrm{ergs} / \mathrm{sec}$. The signal from the direction of $3 \mathrm{C} 273$ would be $1.8 \times 10^{-10} \mathrm{ergs} \mathrm{cm}^{-2} \mathrm{sec}^{-1}$. If we place it at $500 \mathrm{mpc}$, the luminosity would be $5.5 \times 10^{45} \mathrm{ergs} / \mathrm{sec}$.

F.J. Low: What is the certainty of your identification of 3 C 273? In other words, what is the chance that one of these other sources you showed might be somewhere in a fan beam?

H. Friedman: I think the answer is evident in the data I showed. In the scan direction the source appears in the scan field within a tenth degree of the known position of $3 \mathrm{C} 273$. If it were displaced at right angles to the center of the scan pattern then the flux would decrease according to the triangular pattern. If it were $4^{\circ}$ off, the observed flux would be down a factor of 2 . If it were off much more than $4^{\circ}$, its true flux would be so great that it would have been observed at the displaced position in earlier surveys of lesser sensitivity. 